\title{
A preliminary study of in vitro and in vivo synergistic effects of ciprofloxacin and D-tyrosine against Pseudomonas aeruginosa isolates
}

\author{
Huirong $\mathrm{Li}^{+}$, Wei Jiang ${ }^{+}$, Xiaoshuang He*, Mengting Chen* \\ Department of Rheumatology, Immunology and Nephrology, The Central Hospital of Wuhan, Tongji Medical College, Huazhong \\ University of Science and Technology, Wuhan 430014, China
}

*For correspondence: Email: xiaoshuanghe2019@outlook.com; mengtingcheng2019@outlook.com; Tel/Fax: (86) 2782211488; (86) 27-82811446

Sent for review: 29 March 2020

Revised accepted: 26 July 2020

\begin{abstract}
Purpose: To investigate the synergistic antimicrobial effects of ciprofloxacin and D-tyrosine against drug-resistant bacteria.

Method: The antimicrobial effects of ciprofloxacin and D-tyrosine on clinical isolates of multidrugresistant (MDR) Pseudomonas aeruginosa ( $P$. aeruginosa) no. 3556 were determined in vitro based on time-kill curve, and in vivo in P. aeruginosa-zebrafish infection model. Furthermore, 30 clinical isolates of multidrug-resistant $P$. aeruginosa were used in vitro to ascertain the synergistic effect of the two agents.

Results: Combined use of ciprofloxacin and D-tyrosine produced synergistic effects against the clinical isolate of $P$. aeruginosa no. 3556 in vitro and in vivo. Synergism occurred in $96.67 \%(95 \% \mathrm{Cl}$, range $83.33-99.41 \%)$ of the clinical isolates, and ciprofloxacin dose was reduced in $90 \%(95 \% \mathrm{Cl}$, range $74.38-96.54 \%$ ) of the clinical isolates in vitro.

Conclusion: These preliminary results suggest that the combination of ciprofloxacin and D-tyrosine is a promising therapeutic strategy against MDR P. aeruginosa infections.
\end{abstract}

Keywords: Ciprofloxacin, D-tyrosine, Synergistic, P. aeruginosa, Zebrafish infection model, Time-killing curve

\begin{abstract}
This is an Open Access article that uses a fund-ing model which does not charge readers or their institutions for access and distributed under the terms of the Creative Commons Attribution License (http://creativecommons.org/licenses/by/4.0) and the Budapest Open Access Initiative (http://www.budapestopenaccessinitiative.org/read), which permit unrestricted use, distribution, and reproduction in any medium, provided the original work is properly credited.
\end{abstract}

Tropical Journal of Pharmaceutical Research is indexed by Science Citation Index (SciSearch), Scopus, International Pharmaceutical Abstract, Chemical Abstracts, Embase, Index Copernicus, EBSCO, African Index Medicus, JournalSeek, Journal Citation Reports/Science Edition, Directory of Open Access Journals (DOAJ), African Journal Online, Bioline International, Open-J-Gate and Pharmacy Abstracts

\section{INTRODUCTION}

Pseudomonas aeruginosa has become an increasingly notorious opportunistic pathogen in common bacterial infections, including community-acquired, healthcare-associated, and nosocomial infections [1]. The emergence of multidrug-resistant $P$. aeruginosa, including those resistant to broad-spectrum cephalosporins and carbapenems, has increased dramatically over the last two decades, and it is now a global health problem [1]. It is estimated that $P$. aeruginosa accounts for 5 to $10 \%$ of infections in developing countries, $25 \%$ of which are caused by multidrug-resistant $P$. aeruginosa [2]. Studies have shown that the mortality due to in-hospital infection caused by multidrugresistant $P$. aeruginosa may be up to $31.1 \%$ [2]. 
A major problem associated with antibiotic resistance is that pre-existing antibiotics are rapidly becoming ineffective against new and emerging antibiotic-resistant bacteria [3]. For these reasons, there is need to evolve novel and improved treatments against drug-resistant $P$. aeruginosa infections.

One promising solution is based on the use of combination of two or more conventional antimicrobial agents. This produces increased antimicrobial effects when compared with a single agent. Synergistic effect could offer a potential benefit for treatment of bacterial infections through drug combinations [4]. Many researchers have demonstrated that drug combinations produce synergistic effects. Examples are the use of vancomycin and $\beta$ lactams against Staphylococcus aureus highly resistant to vancomycin, and the use of ciprofloxacin-tobramycin combination against $P$. aeruginosa $[5,6]$. Moreover, the combination of a chemical compound and a conventional antimicrobial agent results in increased antibacterial effects, for example, silver nanoparticles in combination with antibiotics against gram-positive and gram-negative bacterial pathogens $[7,8]$. D-Amino acids have been found to affect biofilm formation. Studies have demonstrated that combination of $D$-amino acids and antimicrobial agents produces synergistic antimicrobial effects against pathogens [9-12]. However, studies on the effect of D-tyrosine as an antimicrobial enhancer against bacteria have resulted in controversial outcomes [12-14].

This study was aimed at determination of the synergistic antimicrobial effects of ciprofloxacin D-tyrosine combination on clinically isolated $P$. aeruginosa No. 3556 in vitro based on time-kill curve, and in vivo in $P$. aeruginosa-zebrafish infection model.

\section{EXPERIMENTAL}

\section{Bacterial strains, culture and preparation}

Thirty-one clinical isolates of $P$. aeruginosa strains used in this study were kept in our laboratory. Multidrug-resistant $P$. aeruginosa strain No. 3556 selected from the above strains was used as reference strain in vitro based on time-kill curve, and in vivo in $P$. aeruginosazebrafish infection model. The strain was inoculated in Luria-Bertani (LB) agar plates, followed by overnight incubation at $37^{\circ} \mathrm{C}$. Then, a single colony on the plate was inoculated into 5-mL fresh LB broth and incubated, with shaking at $180 \mathrm{rpm}$, for $12 \mathrm{~h}$ at $37^{\circ} \mathrm{C}$. Bacterial suspension $(500 \mu \mathrm{L})$ was inoculated into $5 \mathrm{~mL}$ fresh LB broth, followed by incubation at $37^{\circ} \mathrm{C}$, with shaking at $180 \mathrm{rpm}$, for $6 \mathrm{~h}$. The bacterial cultures were subjected to centrifugation at 6,000 $\times \mathrm{g}$ for $5 \mathrm{~min}$. The harvested cell pellets were washed twice with phosphate buffered saline (PBS, pH 7.2) and serially diluted for use in this study.

\section{Antibiotic and D-tyrosine preparation}

Ciprofloxacin was purchased from Sigma-Aldrich (St. Louis, MO, United States). It was dissolved in sterile water to a concentration of $256 \mu \mathrm{g} / \mathrm{mL}$. The drug susceptibility of $P$. aeruginosa strain No. 3556 was chosen according to the Clinical and Laboratory Standards Institute (Clinical and Laboratory Standards Institute (CLSI), 2019) [15]. D-Tyrosine was purchased from SigmaAldrich (St. Louis, MO, United States), and was dissolved in PBS, followed by filtration through $0.22 \mu \mathrm{m}$ membrane filters before use.

\section{Optimization of D-tyrosine concentration}

Pseudomonas aeruginosa No. 3556 was prepared in the same manner as described above. The concentration of D-tyrosine was optimized by co-culturing of $100 \mu \mathrm{L}$ of bacterial suspension and $100 \mu \mathrm{L}$ of serially diluted D-tyrosine (0 to $16 \mu \mathrm{M}$ ) in a 96-well plate. Eight hours later, the $\mathrm{OD}_{600}$ was read in a microplate reader. The experiment was done in 3 replicates. Bacteria culture without D-tyrosine was set as control.

\section{In vitro time-kill curves for single and combination drugs}

The effectiveness of ciprofloxacin and D-tyrosine, alone and in combination, against clinical isolate of multidrug-resistant $P$. aeruginosa No. 3556 was determined using the time-kill curve method. Time-kill assays were carried out according to the CLSI guidelines. In these assays, $5 \times 10^{5}$ $\mathrm{CFU} / \mathrm{mL}$ inoculum was incubated in 96-well plate containing adjusted Mueller Hinton broth supplemented with ciprofloxacin and D-tyrosine, alone or in combination, in separate wells. In the entire experiment, the concentration of ciprofloxacin varied from 16 to $0.5 \mu \mathrm{g} / \mathrm{mL}$, while the concentration of D-tyrosine was kept at $8 \mu \mathrm{M}$, based on the optimization result. Aliquots were removed from each tube and diluted serially (1:10) with PBS for use in determination of cell viability after $8 \mathrm{~h}$ of culture in Luria-Bertani (LB) agar plates. Time-kill experiment was performed in triplicate. According to a previous description [16], a combination of two antimicrobial agents was considered synergistic if it caused $a \geq 2 \log$ 
unit reduction in $\mathrm{CFU} / \mathrm{mL}$, relative to the sum of the reductions observed with the individual compounds at the end of the experiment.

Evaluation of the effectiveness of ciprofloxacin and D-tyrosine, in combination and singly, against clinical isolate of $P$. aeruginosa

The degrees of effectiveness of ciprofloxacin and D-tyrosine, alone and in combination, against 30 clinical isolates of $P$. aeruginosa in vitro were determined based on the procedure of time-kill curve for multiple-drug-resistant $P$. aeruginosa No. 3556. The concentration of ciprofloxacin varied from $32 \mu \mathrm{g} / \mathrm{mL}$ to $0.06 \mu \mathrm{g} / \mathrm{mL}$.

In vivo assessment of the effectiveness of ciprofloxacin and D-tyrosine in combination and singly, on $\boldsymbol{P}$. aeruginosa-zebrafish infection model

Each zebrafish was infected with $10 \mu \mathrm{L}$ of a $5 \times$ $10^{5} \mathrm{CFU} / \mathrm{ml}$ bacterial suspension in the treatment group ( $n=10$ per group). The treatment groups were exposed to ciprofloxacin $(8 \mu \mathrm{g} / \mathrm{mL})$ and Dtyrosine $(8 \mu \mathrm{M})$, singly and in combination. Zebrafish without bacteria served as the control group. The percentage survival in each experimental group was monitored from $0 \mathrm{~h}$ to $144 \mathrm{~h}$ post-infection.

\section{Statistical analysis}

Results from in vitro and in vivo experiments are presented as mean \pm standard deviation (SD). GraphPad InStat version 6.01 (GraphPad Software, CA, USA) was used for statistical analysis of the differences amongst group means, based on one-way analysis of variance (ANOVA). Differences were assumed statistically significant at $p<0.05$.

\section{RESULTS}

\section{Optimized D-tyrosine concentration}

The results showed that D-tyrosine interfered with the formation of biofilm, as shown in Figure 1 A. The formation of $P$. aeruginosa No. 3556 biofilm decreased with increase in the concentration of D-tyrosine from 0 to $8 \mu \mathrm{M}$, and subsequent microplate reader results showed that the bacterial concentrations were significantly decreased $(p=0.01<0.05$ ). When $D$-tyrosine concentration was increased to 16 $\mu \mathrm{M}$, there was no change in the formation of $P$. aeruginosa biofilm. Subsequent microplate reader results showed that there was no significant change in the bacterial concentration
( $p=0.23>0.05)$. Thus, $8 \mu \mathrm{M}$ D-tyrosine was selected as the optimum concentration used for investigating the effects of ciprofloxacin and Dtyrosine on clinical isolates of $P$. aeruginosa No. 3556 in vitro and in vivo.
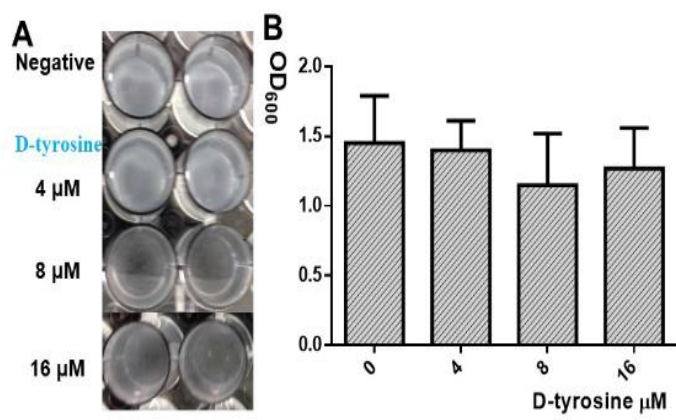

Figure 1: Optimization of D-tyrosine concentration. Effect of D-tyrosine on the formation of $P$. aeruginosa biofilm in 96-well plates (A), and comparison of bacteria growth in the presence or absence of $D$ tyrosine at various concentrations for $8 \mathrm{~h}(\mathrm{~B})$.

\section{Single and combined effects of ciprofloxacin and D-tyrosine in vitro based on time-kill curve test}

The antimicrobial effects of ciprofloxacin and Dtyrosine, singly and in combination, were determined based on the growth properties of $P$. aeruginosa No. 3556. As shown in Figure 2, the bacteria growth when treated with ciprofloxacin or D-tyrosine alone was set as control group, and the results indicated that $P$. aeruginosa No. 3556 was resistant to both agents. In contrast, the combination of ciprofloxacin and D-tyrosine reduced the bacteria from $10 \log \mathrm{CFU} / \mathrm{mL}$ to 6 log CFU/mL when the concentration of ciprofloxacin used ranged from 8 to $16 \mu \mathrm{M}$. The reduction margin in bacteria (4 $\log \mathrm{CFU} / \mathrm{mL}$ ) indicated that the ciprofloxacin - D-tyrosine combination resulted in synergistic/additive effect in vitro. Obviously, the dose of ciprofloxacin could be decreased during combination of ciprofloxacin and D-tyrosine, indicating that the combination was better at killing drug-resistant bacteria than any of the agents when used alone.

\section{Effectiveness of ciprofloxacin and D-tyrosine, alone and in combination, on clinical isolates of $P$. aeruginosa in vitro}

As shown in Table 1, the degree of synergistic effect for combined use of ciprofloxacin and Dtyrosine was $96.67 \%$ (95 \% Cl: 83.33 - 99.41). For ciprofloxacin-resistant strains, the degree of synergistic effect was $95.24 \%(95 \%$ Cl: 77.33 99.15), while for ciprofloxacin-susceptible strain, the degree of synergistic effect was $100 \%$ (95\% $\mathrm{Cl}: 70.09$ - 100). Moreover, the concentrations of 
ciprofloxacin for $90 \%$ (95 \% Cl: 74.38 - 96.54) of the strains were reduced, when compared with ciprofloxacin and D-tyrosine, in combination and singly, against clinical isolates of $P$. aeruginosa in vitro.

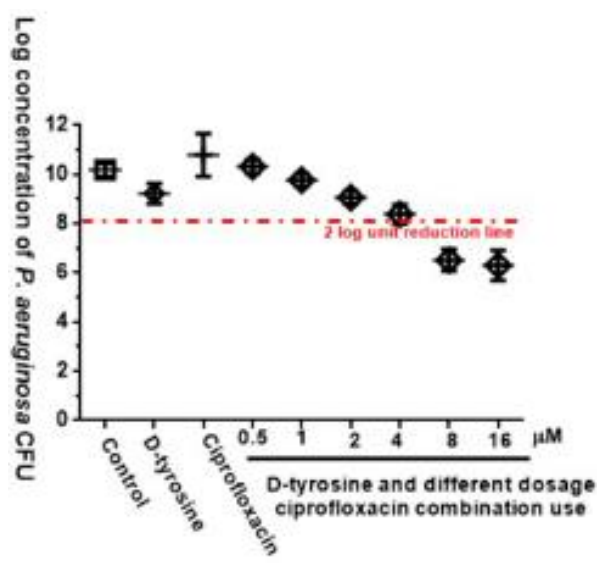

Figure 2: In vitro effect of ciprofloxacin and D-tyrosine on $P$. aeruginosa, singly and in combination, based on time-kill curve test

\section{Effect of ciprofloxacin and D-tyrosine on $P$. aeruginosa-zebra fish infection model}

As shown in Figure 3, the survival of untreated zebrafish was $100 \%$ (from 0 to $144 \mathrm{~h}$ ). This served as control. In the group injected with $P$. aeruginosa No. 3556, the survival of zebrafish was $10 \%$ in $24 \mathrm{~h}$, with no survival in $144 \mathrm{~h}$. The percentage survival of zebrafish was $40 \%$ in 24 h, $20 \%$ in $48 \mathrm{~h}$, and $20 \%$ in $144 \mathrm{~h}$ for the group injected with $P$. aeruginosa No. 3556 and ciprofloxacin. In the group injected with $P$. aeruginosa No. 3556 and D-tyrosine, the survival of zebrafish was $20 \%$ in $24 \mathrm{~h}, 10 \%$ in $48 \mathrm{~h}$, and $10 \%$ in $144 \mathrm{~h}$. In contrast, the survival of zebrafish was $70 \%$ in $24 \mathrm{~h}, 50 \%$ in $48 \mathrm{~h}$ and 40 $\%$ in $144 \mathrm{~h}$ in the group injected with $P$. aeruginosa No. 3556, ciprofloxacin and Dtyrosine. Obviously, ciprofloxacin and D-tyrosine improved the survival of zebrafish and produced good effect in vivo.

Table 1: Antimicrobial effectiveness of ciprofloxacin and D-tyrosine, alone and in combination, against clinical isolates of $P$. aeruginosa

\begin{tabular}{|c|c|c|c|c|c|}
\hline Strain & $\mathrm{MIC} / \mathrm{CIP}$ & R/S & $\begin{array}{l}\text { Effective CIP concentration } \\
\text { in combination use }\end{array}$ & $\begin{array}{l}\text { Log unit reduction in } \\
\text { CFU/mL }\end{array}$ & $\begin{array}{l}\text { Synergism } \\
\text { Yes or No }\end{array}$ \\
\hline No.2241 & 8 & $\mathrm{R}$ & 4 & $4.50 \pm 0.14$ & Yes \\
\hline No.2002 & 0.25 & S & 0.06 & $5.70 \pm 0.00$ & Yes \\
\hline No.3130 & 16 & $\mathrm{R}$ & 4 & $3.92 \pm 0.41$ & Yes \\
\hline No.3361 & 2 & $\mathrm{R}$ & 0.5 & $4.11 \pm 0.33$ & Yes \\
\hline No.2781 & 2 & $\mathrm{R}$ & 2 & $2.94 \pm 0.27$ & Yes \\
\hline No.2302 & 4 & $\mathrm{R}$ & 2 & $3.06 \pm 0.06$ & Yes \\
\hline No.2987 & 32 & $\mathrm{R}$ & 8 & $4.95 \pm 0.23$ & Yes \\
\hline No.3389 & 16 & $\mathrm{R}$ & 8 & $3.01 \pm 0.19$ & Yes \\
\hline No.2904 & 0.5 & $S$ & 0.12 & $2.75 \pm 0.09$ & Yes \\
\hline No.3103 & 0.5 & $S$ & 0.25 & $3.88 \pm 0.69$ & Yes \\
\hline No.3867 & 16 & $\mathrm{R}$ & 4 & $3.42 \pm 0.52$ & Yes \\
\hline No.3402 & 4 & $\mathrm{R}$ & 2 & $2.79 \pm 0.37$ & Yes \\
\hline No. 2725 & 2 & $\mathrm{R}$ & 0.25 & $5.00 \pm 0.45$ & Yes \\
\hline No.2241 & 4 & $\mathrm{R}$ & 1 & $4.16 \pm 0.03$ & Yes \\
\hline No.2321 & 8 & $\mathrm{R}$ & 0.5 & $3.00 \pm 0.14$ & Yes \\
\hline No.3308 & 1 & $S$ & 0.5 & $5.70 \pm 0.00$ & Yes \\
\hline No.3352 & 0.25 & $S$ & 0.25 & $2.41 \pm 0.10$ & Yes \\
\hline No.3404 & 0.5 & $S$ & 0.25 & $2.50 \pm 0.11$ & Yes \\
\hline No.2988 & 2 & $\mathrm{R}$ & 0.5 & $4.20 \pm 0.53$ & Yes \\
\hline No.3065 & 32 & $\mathbf{R}$ & 32 & $1.07 \pm 0.27$ & No \\
\hline No.3321 & 8 & $\mathrm{R}$ & 4 & $5.13 \pm 0.14$ & Yes \\
\hline No.2644 & 4 & $\mathrm{R}$ & 0.5 & $2.98 \pm 0.22$ & Yes \\
\hline No.2241 & 8 & $\mathrm{R}$ & 1 & $4.44 \pm 0.48$ & Yes \\
\hline No.2241 & 0.5 & $S$ & 0.12 & $3.88 \pm 0.13$ & Yes \\
\hline No.3901 & 4 & $\mathrm{R}$ & 1 & $4.61 \pm 0.21$ & Yes \\
\hline No.3875 & 4 & $\mathrm{R}$ & 2 & $3.09 \pm 0.60$ & Yes \\
\hline No.2802 & 16 & $\mathrm{R}$ & 4 & $4.65 \pm 0.07$ & Yes \\
\hline No.3658 & 0.25 & S & 0.06 & $4.00 \pm 0.32$ & Yes \\
\hline No.3716 & 0.5 & $S$ & 0.25 & $3.78 \pm 0.41$ & Yes \\
\hline No.2066 & 2 & $\mathrm{R}$ & 0.5 & $4.33 \pm 0.02$ & Yes \\
\hline
\end{tabular}




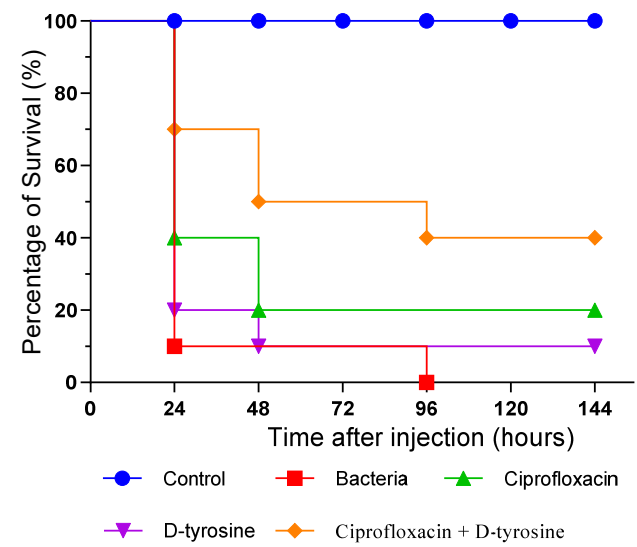

Figure 3: Effects of ciprofloxacin and D-tyrosine, singly and in combination, on zebrafish model of $P$. aeruginosa infection. Control = zebrafish alone, bacteria = zebrafish infected by bacteria, ciprofloxacin = zebrafish infected with bacteria and treated with ciprofloxacin alone, D-tyrosine = zebrafish infected with bacteria and treated with $\mathrm{D}$-tyrosine alone, and ciprofloxacin + D-tyrosine $=$ zebrafish infected with bacteria and treated with combination of ciprofloxacin and D-tyrosine

\section{DISCUSSION}

Bacterial resistance has always been a serious problem. Combined treatment with multiple drugs or chemical compounds may be a solution to the problem of drug resistance. In this preliminarily study, the effects of ciprofloxacin and D-tyrosine, singly and in combination, on multidrug-resistant $P$. aeruginosa were investigated in vitro and in vivo. D-Tyrosine is a bioactive substance. However, the report that Damino acids may inhibit biofilms has remained controversial $[13,14,17]$. The results of the present study showed that D-tyrosine did not completely prevent the formation of biofilm, but inhibited the growth of bacteria by influencing the formation of biofilm to some extent. This might explain the synergistic effect of ciprofloxacin and D-tyrosine against the clinical isolates of $P$. aeruginosa in vitro and in vivo.

Time-kill curve results showed that combination of ciprofloxacin and D-tyrosine produced synergistic effect against $P$. aeruginosa in vitro. As explained above, it might be that D-tyrosine reduced the bacteria biofilm formation and accelerated the entry of ciprofloxacin into the cell, thereby increasing the efficacy of the drug. Furthermore, the use of D-tyrosine reduced the dose of ciprofloxacin in the time-kill curve. The decrease in drug dose during treatment could reduce the risk of drug-resistance. The combination of ciprofloxacin and D-tyrosine improved the survival time of zebrafish in $P$. aeruginosa-zebrafish infection model experiment. This result indicates that the synergistic effect seen in vitro was also produced in vivo.

\section{Limitations of the study}

This study has some limitations. Although it has demonstrated that combination of ciprofloxacin and D-tyrosine produced positive synergistic effect in vitro and in vivo in time-kill curve and $P$. aeruginosa-zebrafish infection model, respectively, single MDR $P$. aeruginosa is not sufficient as an index strain for a viable study. More MDR bacterial strains should be investigated in future in order to determine the precise mechanism involved in the observed synergism.

\section{CONCLUSION}

The findings of this study suggest that the ciprofloxacin/D-tyrosine combination produces synergistic effect in vitro and in vivo. This phenomenon offers a new strategy for tackling the current problem of drug-resistant bacteria.

\section{DECLARATIONS}

\section{Conflict of interest}

No conflict of interest is associated with this work.

\section{Contribution of authors}

We declare that this work was done by the authors named in this article, and all liabilities pertaining to claims relating to the content of this article will be borne by them. Huirong Li and Wei Jiang contributed equally to this work.

\section{Open Access}

This is an Open Access article that uses a funding model which does not charge readers or their institutions for access and distributed under the terms of the Creative Commons Attribution License (http://creativecommons.org/licenses/by/ 4.0) and the Budapest Open Access Initiative (http://www.budapestopenaccessinitiative.org/rea d), which permit unrestricted use, distribution, and reproduction in any medium, provided the original work is properly credited.

\section{REFERENCES}

1. Ventola CL. The antibiotic resistance crisis: part 1: causes and threats. P\&T. 2015; 40(4): 277-283. 
2. Lim C, Takahashi E, Hongsuwan M, Wuthiekanun V, Thamlikitkul V, Hinjoy S, Day NP, Peacock SJ, Limmathurotsakul D. Epidemiology and burden of multidrug-resistant bacterial infection in a developing country. elife. 2016; 5: e18082.

3. Ara J, Tian D, Juhee A. Synergistic antimicrobial activity of bacteriophages and antibiotics against Staphylococcus aureus. Food Sci Biotechnol. 2016; 25(3): 935-940.

4. Coronado-Álvare NM, Parra D, Parra-Ruiz J. Clinical efficacy of fosfomycin combinations against a variety of gram-positive cocci. Enferm Infec Micr Cl. 2019; 37(10): 4-10.

5. Climo MW, Patron RL, Archer GL. Combinations of vancomycin and beta-lactams are synergistic against staphylococci with reduced susceptibilities to vancomycin. Antimicrob Agents Ch. 1999; 43(7): 17471753.

6. Devrim D, Metin O. In-vitro efficacy of synergistic antibiotic combinations in multidrug resistant $P$. aeruginosa strains. Yonsei Med J. 2010; 51(1): 111-116.

7. Fayaz AM, Balaji K, Girilal M, Yadav R, Kalaichelvan PT, Venketesan R. Biogenic synthesis of silver nanoparticles and their synergistic effect with antibiotics: a study against gram-positive and gram-negative bacteria. Nanomedicine. 2010; 6(1): 103-109.

8. Surwade P, Ghildyal C, Weikel C, Luxton T, Peloquin D, Fan $X$, Shah V. Augmented antibacterial activity of ampicillin with silver nanoparticles against methicillinresistant Staphylococcus aureus (MRSA). J Antibiot. 2019; 72(1): 50-53.

9. Marr AK, Gooderham WJ, Hancock RE. Antibacterial peptides for therapeutic use: obstacles and realistic outlook. Curr Opin Pharmacol. 2006; 6(5): 468-472.
10. Jia R, Li Y, Almahamedh HH, Gu T. Enhanced Biocide Treatments with $D$-amino Acid Mixtures against a Biofilm Consortium from a Water-Cooling Tower. Front Microbiol. 2017; 8:1538.

11. Qi H, Li BS, Wang H, Cai Q, Quan X, Cui YX, Meng WY. Effects of $d$-valine on periodontal or peri-implant pathogens: Porphyromonas gingivalis biofilm. J Periodontol. 2018; 89(3): 303-314.

12. Milenković J, Hrenović J, Goićbarišić I, Tomić $M$, Djonlagić J, Rajić N. Synergistic anti-biofouling effect of Ag-exchanged zeolite and D-Tyrosine on PVC composite against the clinical isolate of Acinetobacter baumannii. Biofouling. 2014; 30(8): 965-973.

13. Kao WT, Frye M, Gagnon P, Vogel JP, Chole R. D-amino acids do not inhibit $P$. aeruginosa biofilm formation. Laryngoscope Investig Otolaryngol. 2017; 2(1): 4-9.

14. Jia $R$, Yang $D, X u D, G u T$. Mitigation of a nitrate reducing $P$. aeruginosabiofilm and anaerobic biocorrosion using ciprofloxacin enhanced by $D$ tyrosine. Sci Rep. 2017; 7(1): 6946.

15. Institute CaLS: Performance Standards for Antimicrobial Susceptibility Testing, 29th Edition. Clinical and Laboratory Standards Institute (CLSI) 2019.

16. Letrado P, Corsini B, Díez-Martínez R, Bustamante N, Yuste JE, García P. Bactericidal synergism between antibiotics and phage endolysin Cpl-711 to kill multidrugresistant pneumococcus. Future Microbiol. 2018; 13(11): 1215-1223.

17. She P, Chen L, Liu H, Zou Y, Luo Z, Koronfel A, Wu Y. The effects of $d$-Tyrosine combined with amikacin on the biofilms of P. aeruginosa. Microb Pathog. 2015; 86: 3844. 